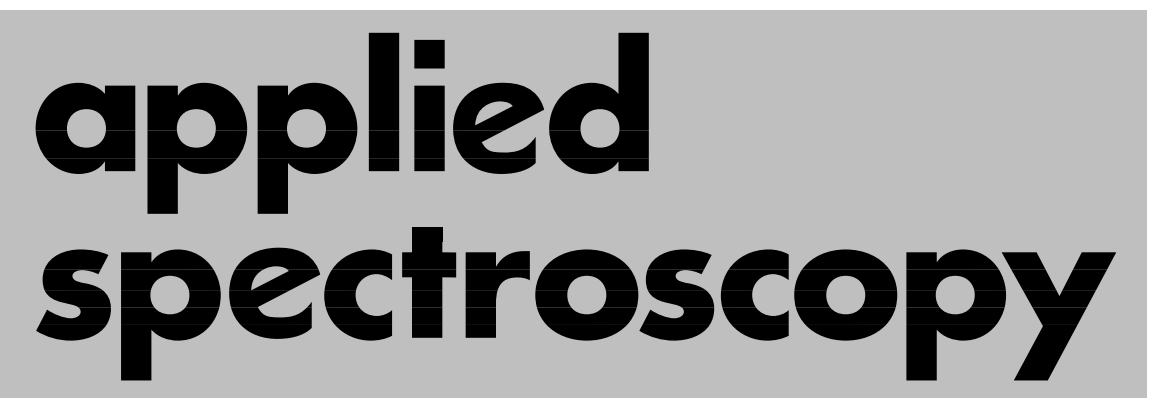

Infrared Spectral Properties of Germ, Pericarp, and Endosperm Sections of Sound Wheat Kernels and Those Damaged by Fusarium graminearum

Kamaranga H. S. Peiris, ${ }^{\text {a } W i l l i a m ~ W . ~ B o c k u s, ~}{ }^{\text {b }}$ Floyd E. Dowell ${ }^{\mathrm{c}} *$

a Department of Biological and Agricultural Engineering, Kansas State University, Manhattan, KS 66502 USA

${ }^{\mathrm{b}}$ Department of Plant Pathology, Kansas State University, Manhattan, KS 66502 USA

c USDA-ARS, CGAHR Engineering and Wind Erosion Research Unit, Manhattan, KS 66502 USA 



\title{
Infrared Spectral Properties of Germ, Pericarp, and Endosperm Sections of Sound Wheat Kernels and Those Damaged by Fusarium graminearum
}

\author{
Kamaranga H. S. Peiris, ${ }^{a}$ William W. Bockus, ${ }^{b}$ Floyd E. Dowell ${ }^{c}, *$ \\ ${ }^{a}$ Department of Biological and Agricultural Engineering, Kansas State University, Manhattan, KS 66502 USA \\ ${ }^{b}$ Department of Plant Pathology, Kansas State University, Manhattan, KS 66502 USA \\ c USDA-ARS, CGAHR Engineering and Wind Erosion Research Unit, Manhattan, KS 66502 USA
}

\begin{abstract}
Mid-infrared attenuated total reflection (Mid-IR-ATR) spectra (4000-380 $\mathrm{cm}^{-1}$ ) of pericarp, germ, and endosperm sections from sound and Fusarium-damaged wheat kernels of cultivars Everest and Tomahawk were collected using a Fourier transform infrared (FT-IR) spectrometer. The differences in infrared absorption bands between sound and Fusarium-damaged kernels were examined. Absorption bands in which differences were identified were compared with the mid-IR-ATR absorption bands of deoxynivalenol (DON) and Fusarium graminearum hyphae. Marked differences in absorption patterns were observed between sound and Fusarium-damaged pericarp and germ spectra, whereas those differences were negligible in the endosperm spectra. Fusarium-damaged pericarp and germ spectra exhibited a shift in the peak position of the band near $1035 \mathrm{~cm}^{-1}$ along with increased absorptions at 1160, 1203, 1313, and $1375 \mathrm{~cm}^{-1}$, likely due to the influence of DON and fungi in the Fusarium-damaged kernel tissue matrix. These results suggest that infrared spectroscopy can detect DON in the surface tissues of Fusarium-damaged wheat kernels.
\end{abstract}

Index Headings: Wheat; Fusarium graminearum; Fusarium head blight; Deoxynivalenol; DON; Attenuated total reflection spectroscopy; ATR spectroscopy; Fourier transform infrared spectroscopy; FT-IR spectroscopy.

\section{INTRODUCTION}

Fusarium head blight (FHB) is a fungal disease complex of wheat and other small grain cereals that can reduce grain yield and quality when warm, humid weather conditions are experienced at the time of flowering. ${ }^{1}$ The food and feed prepared from Fusarium-damaged grains pose a health risk to humans and animals due to the presence of Fusarium mycotoxins..$^{1-3}$ In North America, Fusarium graminearum is the predominant causal agent of FHB, although several other Fusarium species can also cause the disease. ${ }^{4,5}$

The mycotoxins produced by the Fusarium spp. include trichothecenes, zearalenone, moniliformin, fumonisins, and enniatins. ${ }^{6}$ Trichothecenes constitute the largest group of mycotoxins produced by Fusarium spp. in cereal grains. Trichothecenes can be further subdivided into four categories (types A-D) based on the functional groups attached to their tetracyclic 12,13-epoxy trichothecene skeleton. ${ }^{7}$ The type B trichothecenes, which have a carbonyl function at $\mathrm{C} 8$, are the most common and include deoxynivalenol (DON) and the closely related nivalenol. ${ }^{8}$ DON is produced by $F$. graminearum and $F$. culmorum, and nivalenol is produced by several

Received 11 April 2012; accepted 30 May 2012.

* Author to whom correspondence should be sent. E-mail: floyd.dowell@ ars.usda.gov.

DOI: $10.1366 / 11-06683$ isolates of these two species as well as by $F$. poae and $F$. equiseti. ${ }^{9,10}$

Wheat is most susceptible to Fusarium infection at anthesis, although infection can also occur either during the latter stages of kernel development or in mature grains under warm, humid environmental conditions that favor growth of fungus. ${ }^{11}$ Fusarium hyphae enter developing kernels through the brush end, colonize the surface layers, and move inward toward the endosperm. Infected kernels contain DON in both host tissues and fungal hyphae. In hyphal cells, the toxins are localized in the cytoplasm, mitochondria, vacuoles, and cell wall. In the host plant cells, the toxins are detected in the cytoplasm, chloroplasts, plasmalemma, cell wall, and vacuoles. ${ }^{12,13}$ In Fusarium-damaged kernels, fungal hyphae are present on the kernel surface and inside the tissues. Fungal hyphae are most prevalent in aleurone and pericarp tissues but are also found throughout the endosperm. ${ }^{14,15}$ Schnurer $^{16}$ reported that a higher proportion (97\%) of fungal biomass was found in the bran fraction of wheat, which constituted $31 \%$ of the kernel weight. Trigo-stockli et al. ${ }^{17}$ showed that mycotoxin contents were generally highest in the bran and lowest in the flour for milled fractions of wheat. Therefore, DON within a Fusariumdamaged kernel appears to be mostly concentrated in peripheral tissues, both in host cells and in fungal hyphae.

In modern wheat production, the use of FHB-resistant cultivars is considered to be one of the best tools available to manage FHB disease and thereby achieve a lower DON content in harvested grains, which is very important in grain marketing. Wheat breeding programs for developing FHBresistant cultivars evaluate numerous wheat varieties for Fusarium resistance. FHB resistance is often evaluated by measuring the Fusarium-damaged kernel percentages (FDK) and DON levels in grain samples. Grain FDK is usually visually estimated by trained personnel, ${ }^{18}$ whereas bulk grain DON levels are determined by standard laboratory instrumental techniques, such as gas chromatography-mass spectrometry (GC-MS) and high-performance liquid chromatography (HPLC). ${ }^{7}$ FDK is a subjective measurement, and the laboratory techniques for determining DON are time consuming, expensive, and destructive.

Near-infrared spectroscopy (NIRS) is a widely used analytical technique for the quantitative evaluation of NIR active compounds present in food and agricultural products. ${ }^{19}$ Instrumental techniques based on NIRS have also been developed for estimating FDK and their DON levels in wheat. ${ }^{18,20}$ Mid-infrared (mid-IR) spectroscopy has been used to detect fungal infections and the presence of DON in flour samples from sound and Fusarium graminearum-infected corn 
kernels. ${ }^{21}$ The study of mid-IR spectral properties of sound and Fusarium-damaged wheat kernels, DON, and Fusarium graminearum hyphae may facilitate an understanding of the differences in fundamental infrared absorptions between Fusarium-damaged and sound wheat kernels. Such a study will further help to determine whether DON or the fungi in the Fusarium-damaged kernel tissue matrix cause any of the observed changes in mid-IR absorptions. Any differences in the mid-IR absorption may be reflected in the NIR absorption of sound and Fusarium-damaged kernels, making it possible to extract that information from NIR spectra using multivariate calibration techniques. Therefore, the goal of this study was to collect and examine the mid-IR spectra of Fusarium graminearum hyphae and pure DON, as well as wheat pericarp, germ, and endosperm sections from sound and Fusariumdamaged kernels. The objectives of these analyses were to study the mid-IR spectral absorption patterns of the samples examined, to identify mid-IR spectral absorption differences between sound and Fusarium-damaged kernel parts, and to investigate whether those differences could be related to the spectral absorption bands of DON and Fusarium graminearum hyphae.

\section{EXPERIMENTAL METHODS}

Samples and Collection of Spectra. Wheat kernels for this study were collected from grains harvested in an FHB nursery evaluation trial conducted in 2010/2011 under heavy disease pressure. This disease pressure was achieved by applying corn spawn inoculum of isolate Z-3639 (NRRL 29214) of $F$. graminearum to the soil. The spawn produced perithecia of the fungus, which released ascospores during wheat heading. Infections were enhanced by running overhead impulse sprinklers for 3 min every hour from 9:00 p.m. through 6:00 a.m. during grain fill. The plots were harvested with a smallplot combine. The kernels were visually selected from two wheat cultivars: Everest (moderately resistant to FHB) and Tomahawk (susceptible to FHB). Wheat germ, pericarp, and endosperm sections from each of three different visually selected replicates of sound and Fusarium-damaged kernels were dissected under a microscope. The Fusarium-damaged kernels selected were those with moderate Fusarium damage, as evidenced by a slight shrinkage in kernel size, a change in the pericarp color to off-white or beige, and the presence of pinkish-white Fusarium graminearum mycelia on the surface of the kernel. Such moderately infected kernels were used because in severely infected kernels, both germ and endosperm are completely destroyed. The sound kernels were plump brown kernels without any visual Fusarium mycelia present on the kernel surface under microscopic examination.

The wheat germ and sections of the outer pericarp layer were removed using a tungsten needle. The kernels were then bisected transversely, and starchy sections were extracted from the center of the endosperm. The dissected sections were scanned immediately after extraction using the Universal Attenuated Total Reflection (UATR) accessory of the Perkin Elmer Spectrum 400 FT-IR/FT-NIR spectrometer (Perkin Elmer, Waltham, MA) in the $4000-380 \mathrm{~cm}^{-1}$ spectral region. The samples were pressed to the UATR diamond crystal using the pressure arm of the instrument to a force gauge reading of 145-150. The spectrometer conditions used were a resolution of $2 \mathrm{~cm}^{-1}$, a data interval of $0.5 \mathrm{~cm}^{-1}$, and a mirror speed of 0.1 $\mathrm{cm} / \mathrm{s}$, and ten scans were averaged to obtain the final spectrum for each sample without subjection to attenuated total reflection (ATR) correction. Small aggregates of pure deoxynivalenol

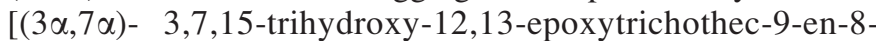
one] (Sigma-Aldrich, St. Louis, MO) crystals were used to collect the spectrum of DON. Fusarium graminearum hyphae were collected from the surfaces of severely infected wheat kernels using tweezers. The DON and Fusarium graminearum hyphae spectra were also collected in triplicate using the UATR accessory and the same spectrometer conditions.

Analysis of Spectra. Absorption spectra collected by the Perkin Elmer instrument in .sp data format were imported and converted to .spc format for further spectral analysis by the GRAMS/AI 8.0 software package (Thermo Electron, Salem, $\mathrm{NH})$. Comparison of bands of absorption spectra with the second-derivative spectra constructed from 9-69 data points at 10-data-point increments showed that use of 49 data points is appropriate to obtain second-derivative spectra reflecting the features of mid-IR absorption bands from data collected at 0.5 $\mathrm{cm}^{-1}$ intervals. Therefore, second derivatives of the absorbance spectra were constructed with 49 data points using the Savitzky-Golay algorithm of the GRAMS software using a second-degree polynomial fit. The spectra from three replicate samples were averaged and used to study differences in mid-IR bands between sound and Fusarium-damaged kernel parts of two cultivars. These differences in spectral band heights and positions were then compared with the mid-IR spectral bands of DON and the Fusarium graminearum hyphae.

\section{RESULTS AND DISCUSSION}

Mid-Infrared Absorption Spectra of Kernel Parts, Fusarium graminearum Hyphae and DON. The mid-IR spectra of a chemical compound provide details of the fundamental vibrations of the groups of its component atoms as well as the specific vibrations of the whole molecule. The mid-IR spectrum of a biological sample is a weighted composite of the spectra of individual chemical compounds present in that sample. This spectrum can therefore provide detailed information about the atomic groups present in the constituent molecules of the sample. The average mid-IR-ATR absorbance spectra $\left(4000-400 \mathrm{~cm}^{-1}\right)$ of three replicate wheat germ, pericarp and endosperm sections collected from sound and Fusarium-damaged kernels of cultivars Everest and Tomahawk are shown in Fig. 1. Significant spectral band differences were not observed among replicate spectra while the slight differences were in relation to the band heights. The wheat kernel parts are composed of numerous chemical compounds such as carbohydrates, proteins, and lipids. Therefore, their respective spectra are composites of the spectra of all those individual compounds present in the kernel tissue, with strongly pronounced spectral features for the major kernel compounds. These spectra and their prominent spectral bands closely resemble the diffuse reflectance mid-IR spectra of ground Hard Red Winter and Durum wheat. ${ }^{22,23}$

The $-\mathrm{OH},-\mathrm{NH}$, and $-\mathrm{CH}$ spectral bands above $2700 \mathrm{~cm}^{-1}$ were relatively similar for all kernel parts (Fig. 1). The spectral bands in the region below $1800 \mathrm{~cm}^{-1}$ were markedly different for the three types of kernel parts. Wheat germ spectra had strong amide I $\left(\sim 1635 \mathrm{~cm}^{-1}\right)$ and amide II $\left(\sim 1540 \mathrm{~cm}^{-1}\right)$ bands in the $1700-1450 \mathrm{~cm}^{-1}$ region. Wheat germ has a higher protein content $(34.1 \mathrm{~g} / 100 \mathrm{~g}$ dry weight) than endosperm (10.2 $\mathrm{g} / 100 \mathrm{~g})$ or pericarp $(5.1 \mathrm{~g} / 100 \mathrm{~g}){ }^{24}$ The amide bands of the 

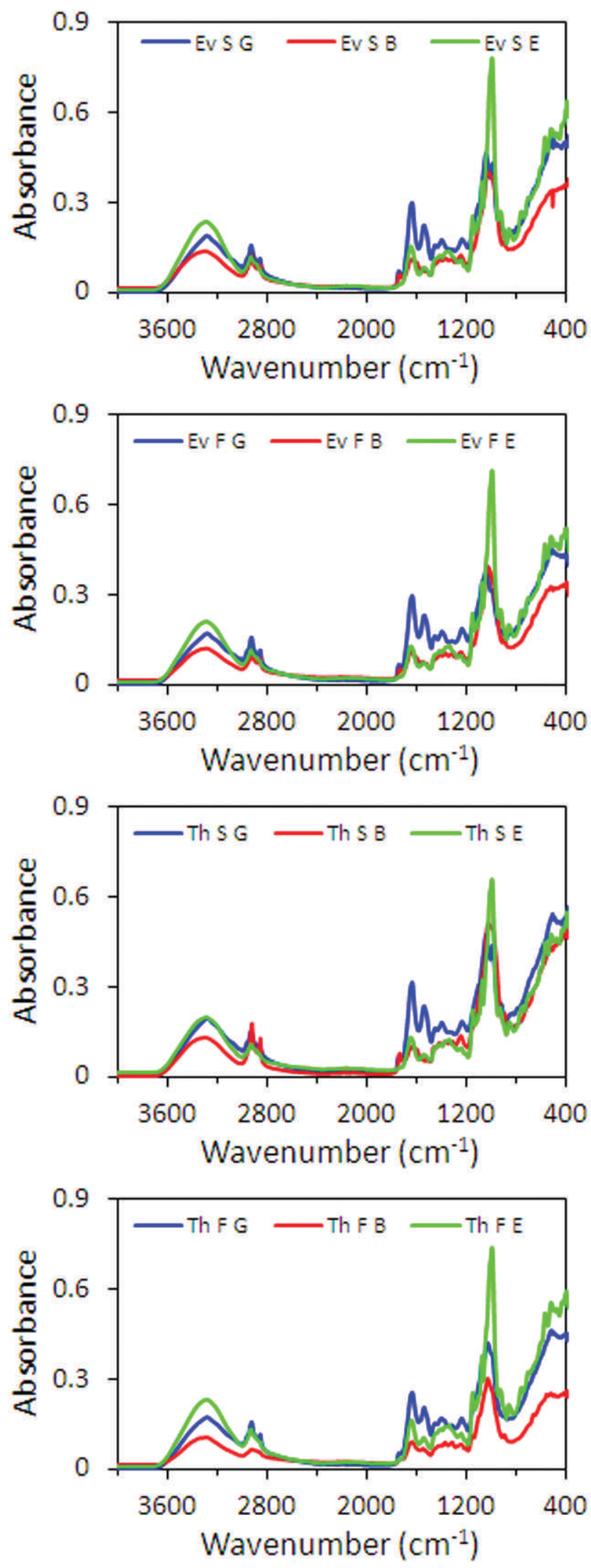

FIG. 1. Mid-infrared absorbance spectra of Everest (Ev) and Tomahawk (Th) wheat germ (G), bran (B), and endosperm (E) sections from sound (S) and Fusarium-damaged (F) wheat kernels. wheat germ spectra are therefore more pronounced than the amide bands of the endosperm or pericarp spectra.

All wheat kernel parts produced a very strong absorption band in the $1200-850 \mathrm{~cm}^{-1}$ region, and this band in the endosperm spectra reached a peak absorbance of above 0.6 units at approximately $995 \mathrm{~cm}^{-1}$. This band may be assigned as representing the $\mathrm{O}-\mathrm{C}$ stretch vibrations of the anhydroglucose ring, which is primarily found in starch but also observed in other carbohydrates, such as cellulose. ${ }^{25}$ The prominence of this band in the wheat endosperm spectra therefore appears to be eminently reasonable, as over $80 \%$ of wheat endosperm carbohydrates are starch. ${ }^{26}$

The mid-IR absorbance spectra of Fusarium graminearum hyphae and DON are shown in Fig. 2. The spectrum of Fusarium graminearum hyphae has a very broad band in the region of $3650-3000 \mathrm{~cm}^{-1}$ with a peak near $3260 \mathrm{~cm}^{-1}$. Barran et al. ${ }^{27}$ showed that the hyphal walls of a Fusarium isolate contained $66.0 \%$ carbohydrate, $7.3 \%$ protein, $5.5 \%$ lipid, and $1.8 \%$ ash. The major cell wall component, $\mathrm{N}$-acetylglucosamine (39\%), was shown to be present as chitin. Therefore, this broad band may be attributed to the stretch vibrations of the $-\mathrm{OH}$ and $-\mathrm{NH}$ groups found in the chitin, other carbohydrates, and proteins of the fungal cell wall. The amide I and II bands $\left(1700-1485 \mathrm{~cm}^{-1}\right)$, which likely arise from fungal proteins, were also prominent in the spectra. The fingerprint regions of the spectra of fungi and the spectra of wheat kernel parts were very smooth and broad, most likely due to the combination of numerous fingerprint bands from the wide variety of chemicals present in the cells of kernel tissues and fungi. However, the fingerprint region of Fusarium graminearum hyphae is dominated by a broad band with a peak near $1035 \mathrm{~cm}^{-1}$. This broad and strong absorption may be produced by fungal carbohydrates. The $\mathrm{C}-\mathrm{O}-\mathrm{C}$ and $\mathrm{C}-\mathrm{C}$ stretch vibrations of carbohydrates are strong and found in this region of the spectrum. ${ }^{28}$

The broad hydroxyl band from $3650-3100 \mathrm{~cm}^{-1}$ in the DON spectrum (Fig. 2) resulted from the stretch vibrations of $-\mathrm{OH}$ groups attached to the $3 \mathrm{C}, 7 \mathrm{C}$, and $15 \mathrm{C}$ of the $\mathrm{DON}$ molecule (Fig. 3). The other group of bands observed from $3030-2800 \mathrm{~cm}^{-1}$ can be assigned to the $\mathrm{CH}$ stretch vibrations of $-\mathrm{CH},-\mathrm{CH}_{2}$, and $-\mathrm{CH}_{3}$ groups, including those that are attached to the C9-C10 double bond. The band with the peak at $1680 \mathrm{~cm}^{-1}$ is due to carbonyl group vibration. ${ }^{29}$ In the fingerprint region of the DON spectra, several sharp major peaks were noted at 1167,1023 , and $947 \mathrm{~cm}^{-1}$. These bands may be assigned to the $\mathrm{C}-\mathrm{O}-\mathrm{C}$ antisymmetric stretch (1167 $\mathrm{cm}^{-1}$ ) and the epoxide ring $\mathrm{C}-\mathrm{O}$ antisymmetric stretch (947 $\mathrm{cm}^{-1}$ ). Reedy and Mossoba ${ }^{30}$ assigned the mid-IR bands of trimethylsilyl derivatives of DON at $1178 \mathrm{~cm}^{-1}$ and $965 \mathrm{~cm}^{-1}$ to the $\mathrm{C}-\mathrm{O}-\mathrm{C}$ antisymmetric stretch and the epoxide ring $\mathrm{C}-\mathrm{O}$ antisymmetric stretch, respectively, but they did not observe the very strong band at $1023 \mathrm{~cm}^{-1}$ that we observed in the ATR-FT-IR spectrum of pure DON.

Spectral Differences Between Sound and FusariumDamaged Wheat Kernels. The absorption of mid-IR radiation and the spectral band peak heights in the ATR spectra are affected by the sample size and the force applied to press the sample to the ATR crystal during scanning. The secondderivative of the spectra minimizes the baseline shifts caused by the sample size and pressure variations often encountered in ATR spectroscopy. The negative peak heights of the secondderivative spectra are proportional to the absorbance peak heights. ${ }^{31,32}$ Therefore, second-derivative spectra were used to 


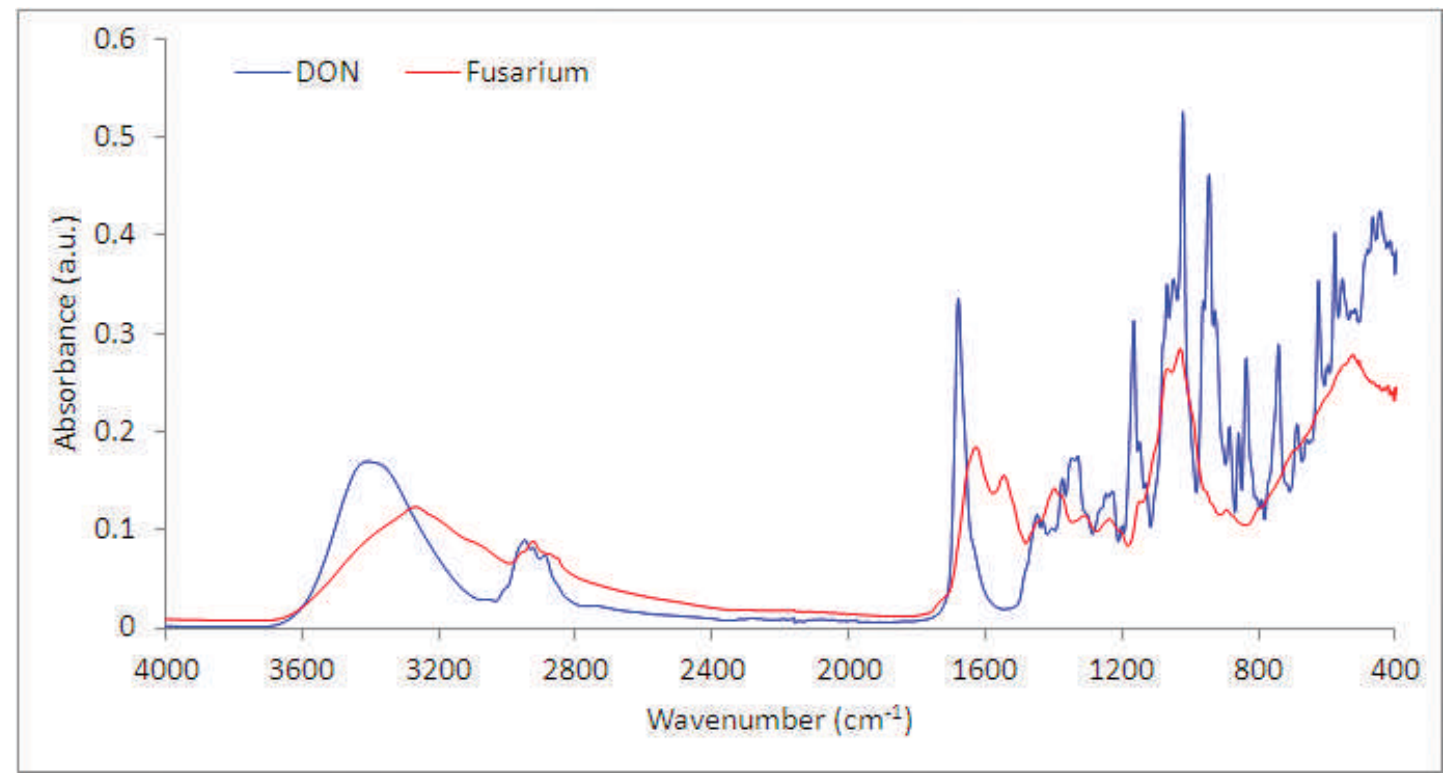

FIG. 2. Mid-infrared absorbance spectra of deoxynivalenol (DON) and Fusarium hyphae.

compare spectral differences between sound and Fusariumdamaged kernel tissue samples.

Most of the variations in spectral bands were noted in the region below $1800 \mathrm{~cm}^{-1}$; thus, second-derivative spectra from sound and Fusarium-damaged endosperm sections of two wheat cultivars are presented for the $1800-600 \mathrm{~cm}^{-1}$ region (Fig. 4). The variation in band peak positions and heights of endosperm spectra are very slight, demonstrating that all spectral bands of sound and Fusarium-damaged endosperm spectra overlap each other. The endosperm sections were extracted from the center of the endosperm, and these results confirm that Fusarium graminearum had not significantly altered the endosperm chemistry in moderately infected kernels. In contrast, considerable variations were noted between sound and Fusarium-damaged pericarp spectra (Fig. 5 ). The peak height of the bands at 1160, 1203, 1313, and 1375 $\mathrm{cm}^{-1}$ were higher for the Fusarium-damaged pericarp of both cultivars, whereas the Tomahawk peaks were higher than the Everest peaks at those wavelengths. The spectral band peak positions of the Fusarium-damaged kernel parts near 1100 $\mathrm{cm}^{-1}$ and $1035 \mathrm{~cm}^{-1}$ showed certain shifts from the band peak positions observed in sound kernels. In particular, Fusariumdamaged pericarp had peaks near $1105 \mathrm{~cm}^{-1}$ and $1032 \mathrm{~cm}^{-1}$, whereas the same bands for sound pericarp were at $1100 \mathrm{~cm}^{-1}$ and $1037 \mathrm{~cm}^{-1}$, respectively, showing a shift of approximately $5 \mathrm{~cm}^{-1}$. Similarly, Fusarium-damaged wheat germ also had elevated band peaks at 1160, 1203, 1313, and $1375 \mathrm{~cm}^{-1}$, though the differences were very small at 1203 and $1313 \mathrm{~cm}^{-1}$ (Fig. 6). Additionally, the peak heights of the bands at 1079 and $955 \mathrm{~cm}^{-1}$ were notably higher in the Fusarium-damaged germ spectra; however, this trend was not observed for the pericarp spectra. A shift in peak height position was noted wherein the spectral band peak at $1043 \mathrm{~cm}^{-1}$ for sound wheat germ spectra of both cultivars was shifted to 1038 and 1030 $\mathrm{cm}^{-1}$ for Fusarium-damaged Everest and Tomahawk germ spectra, respectively. However, the shift in peak height position noted for the pericarp spectra near $1105-1100 \mathrm{~cm}^{-1}$ was not observed in the germ spectra.
The differences in peak height and the shift in peak height position between sound and Fusarium-damaged kernel parts may be caused by either the direct influence of the DON or fungus present in Fusarium-damaged kernel parts or other chemical metabolic changes in tissues resulting from the activity of fungi during the progression of the infection. It is therefore necessary to compare the changes in spectral bands with respect to the mid-IR spectral bands of DON and fungi to determine whether DON or fungi are the cause of those spectral changes. The second-derivative spectra of DON and Fusarium graminearum hyphae are given in Fig. 7.

Comparison of Spectral Differences Between Sound and Fusarium-Damaged Spectra with DON and Fusarium graminearum Spectral Bands. The differences between sound and Fusarium-damaged pericarp second-derivative spectral bands were compared with the bands of the DON spectrum (Figs. 8 and 9). The strongest mid-IR band of the DON

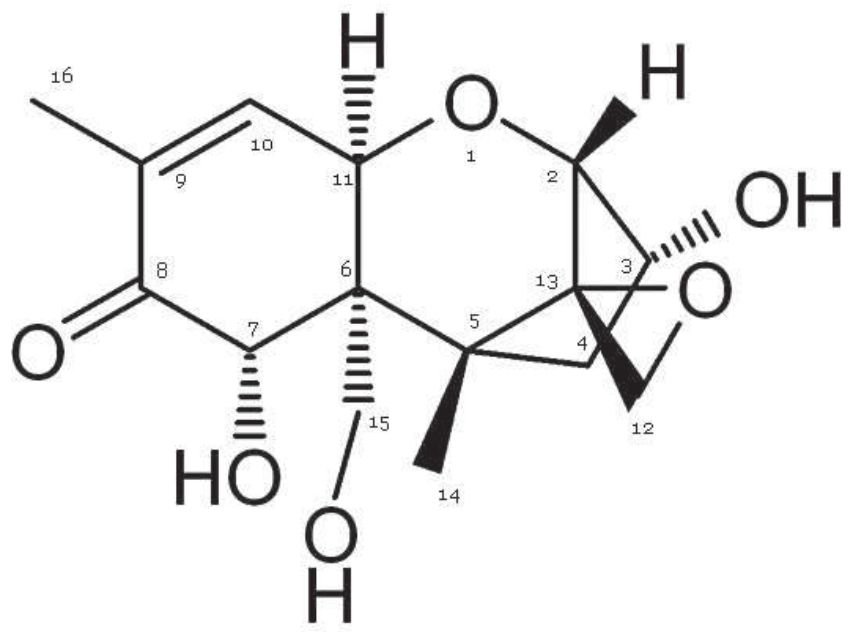

FIG. 3. Chemical structure of DON. 


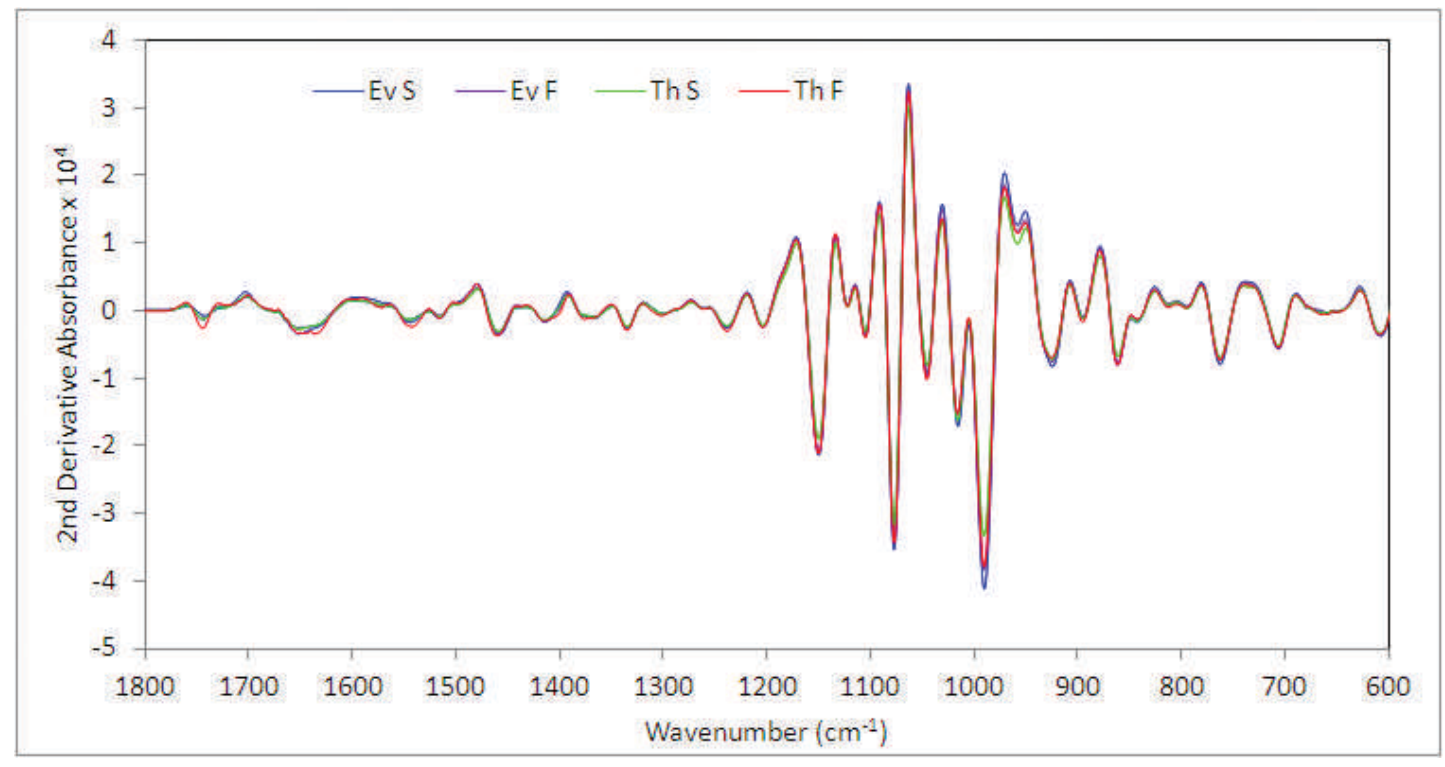

FIG. 4. Second-derivative mid-IR spectra of sound (S) and Fusarium-damaged (F) endosperm sections of Everest (Ev) and Tomahawk (Th) wheat kernels.

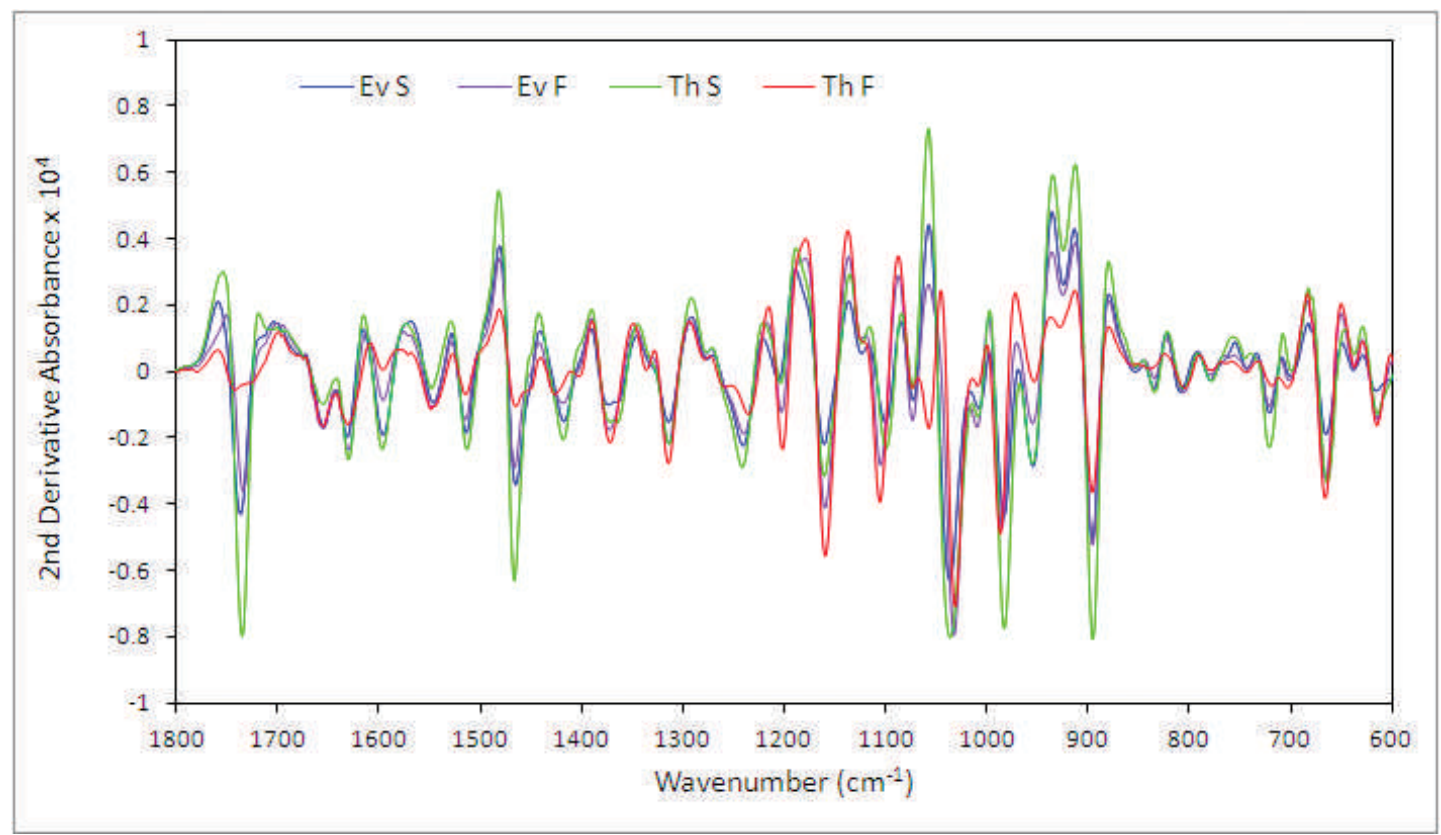

FIG. 5. Second-derivative mid-IR spectra of sound (S) and Fusarium-damaged (F) pericarp sections of Everest (Ev) and Tomahawk (Th) wheat kernels.

spectrum at $1023 \mathrm{~cm}^{-1}$ (Fig. 2) appears to influence the shift of the $1037 \mathrm{~cm}^{-1}$ spectral band of the sound pericarp towards a lower wavenumber in the Fusarium-damaged pericarp spectra (Figs. 8 and 9). Similar spectral band shifts were also observed in the wheat germ spectra (data not shown).

Because the DON spectrum has no particularly strong band near $1105-1100 \mathrm{~cm}^{-1}$, the spectral band peak position shift observed in this area appears to be caused either by the Fusarium graminearum hyphae directly or by another metabolic change in kernel tissue resulting from fungal invasion. The Fusarium graminearum hyphae spectrum showed a stronger absorption near $1110 \mathrm{~cm}^{-1}$ than was observed in the DON spectrum. However, this spectral band shift was not found in the wheat germ spectra. Therefore, this spectral band position shift may not be due to the direct influence of DON or Fusarium graminearum in the wheat tissue matrix.

The increase in the height of the Fusarium-damaged kernel spectral band at $1160 \mathrm{~cm}^{-1}$ appears to be influenced by the DON spectral bands at 1167 and $1150 \mathrm{~cm}^{-1}$ (Figs. 8 and 9). The Fusarium graminearum hyphae spectrum also showed an absorption band at $1152 \mathrm{~cm}^{-1}$ (Fig. 7), which likely reflects the influence of these DON bands as well. Both Fusariumdamaged wheat pericarp and germ spectra (data not shown) 


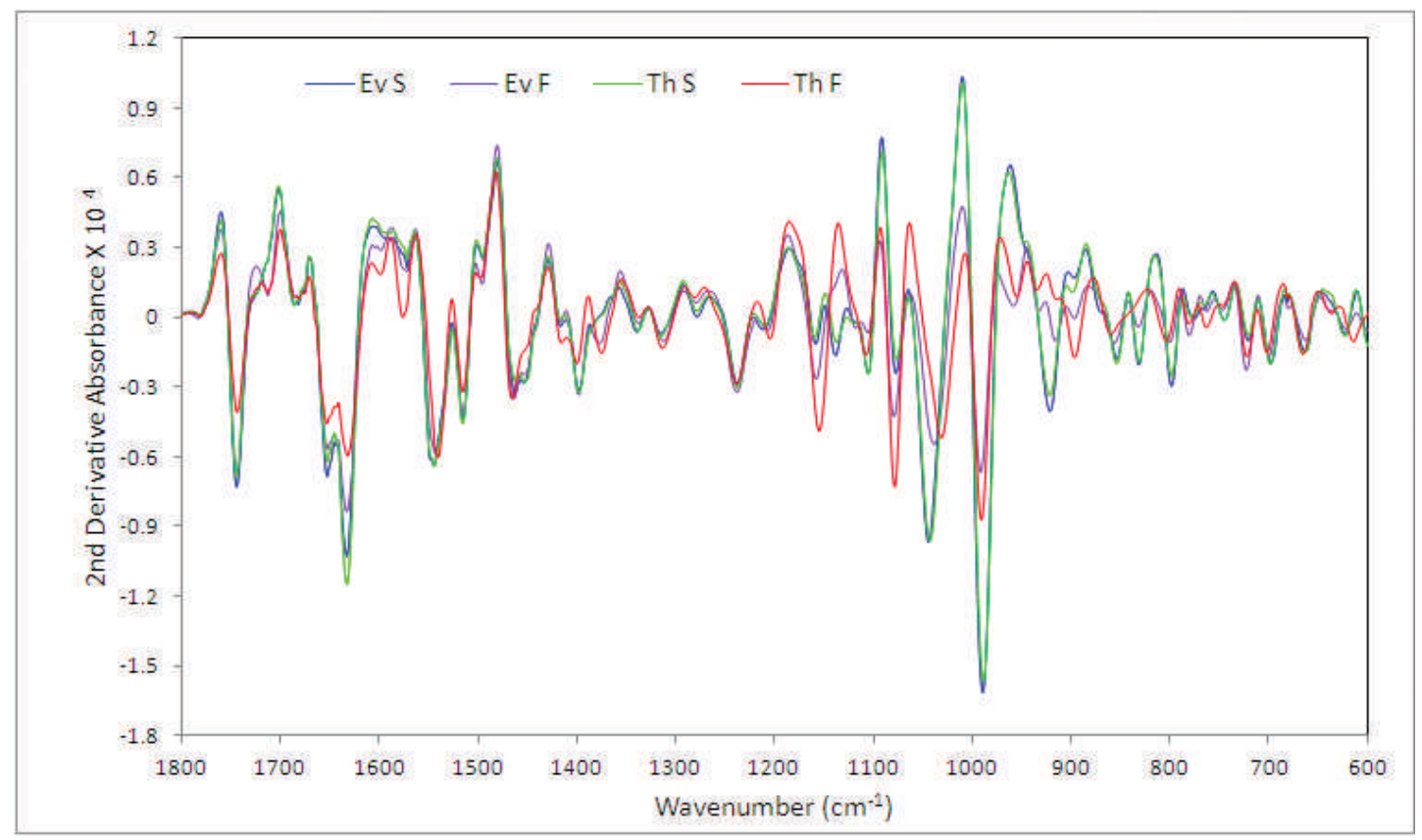

FIG. 6. Second-derivative mid-IR spectra of sound (S) and Fusarium-damaged (F) germ of Everest (Ev) and Tomahawk (Th) wheat kernels.

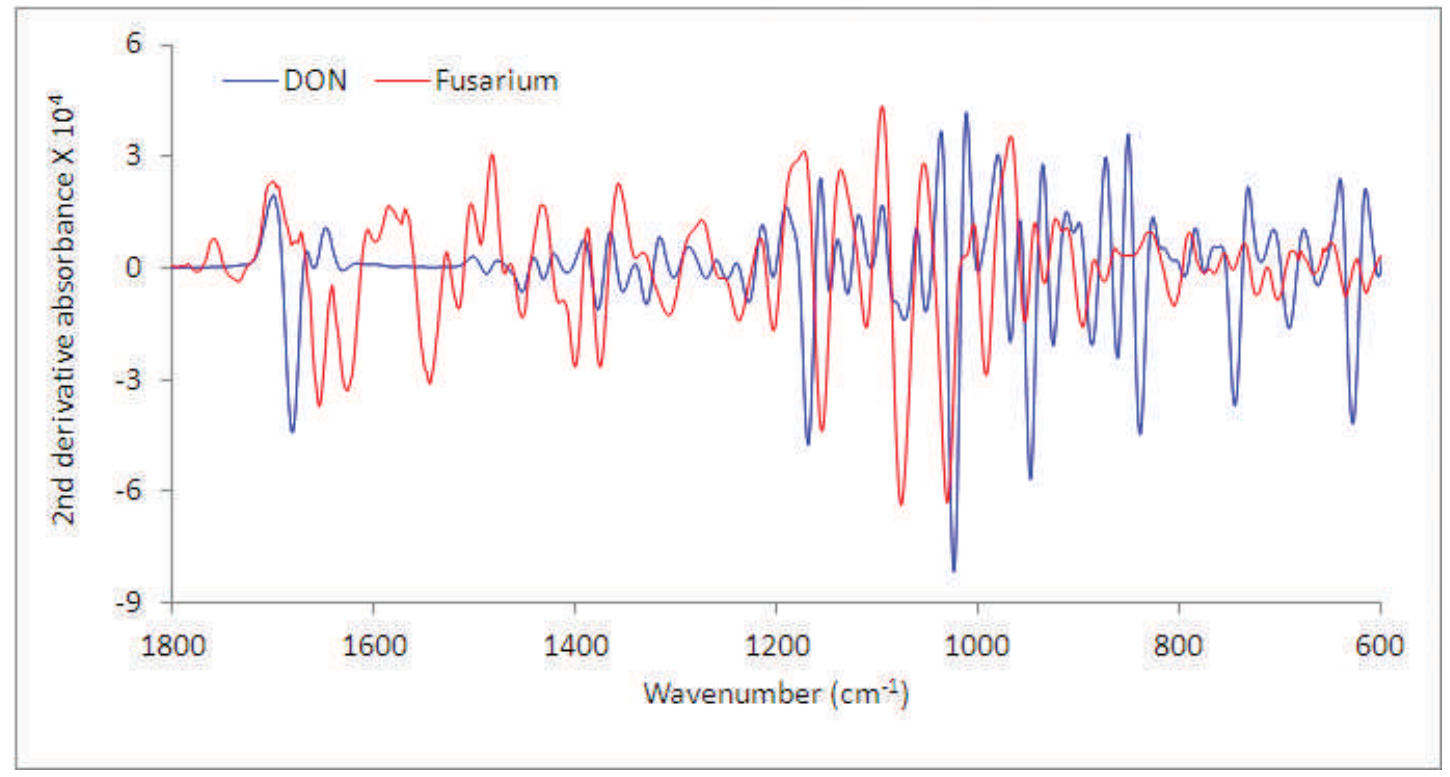

FIG. 7. Second-derivative mid-IR spectra of DON and Fusarium hyphae.

showed similar increases in this band peak height. The Fusarium graminearum hyphae had spectral bands with peaks at 1203,1306 , and $1375 \mathrm{~cm}^{-1}$, whereas DON had bands with peaks at 1203,1301 , and $1375 \mathrm{~cm}^{-1}$. The increase in heights of the spectral bands of Fusarium-damaged kernel pericarp and germ sections observed at 1203,1313 , and $1375 \mathrm{~cm}^{-1}$ may have been influenced by these aforementioned spectral absorptions of DON and fungus.

Mycotoxins in Fusarium-damaged kernels are localized in both the kernel tissue matrix and the fungal hyphae associated with the kernel tissues. ${ }^{12,13}$ In particular, the mycotoxins and fungus are mostly concentrated in the bran layers of the kernel. ${ }^{16,17}$ Thus, it is unsurprising that the fungal hyphae and the DON in the host cells appear to primarily influence the midIR absorption patterns of Fusarium-damaged kernels in these bran layers. Fusarium-damaged single wheat kernels may have DON content varying from undetectable levels to more than 1000 parts per million (ppm). ${ }^{33}$ Because most of the DON is concentrated in the bran tissues, which represent a lower proportion of the kernel weight, the DON concentrations may be several times higher near the surface of the wheat kernel than in the kernel interior. Therefore, infrared absorptions of 


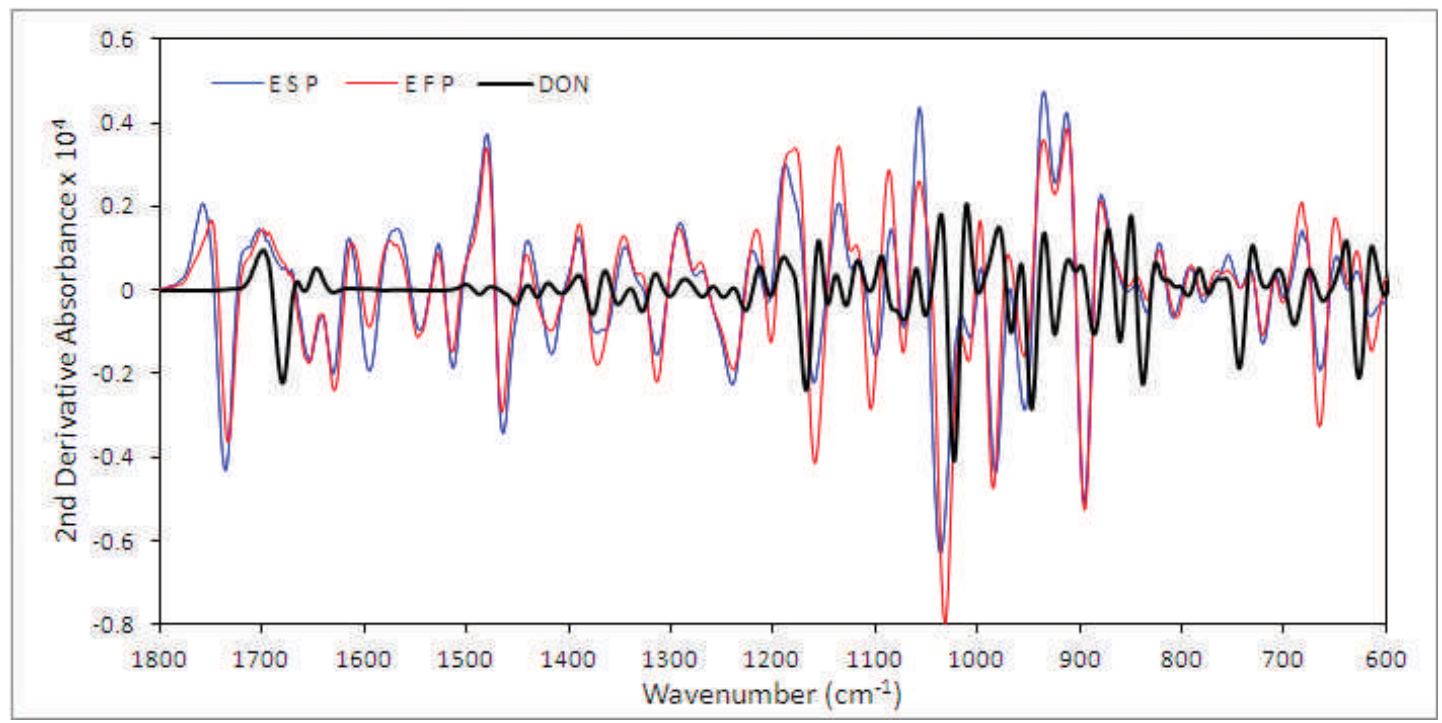

FIG. 8. Second-derivative mid-IR spectra of Everest sound pericarp (ESP) and Fusarium-damaged pericarp (EFP) compared with the DON spectrum.

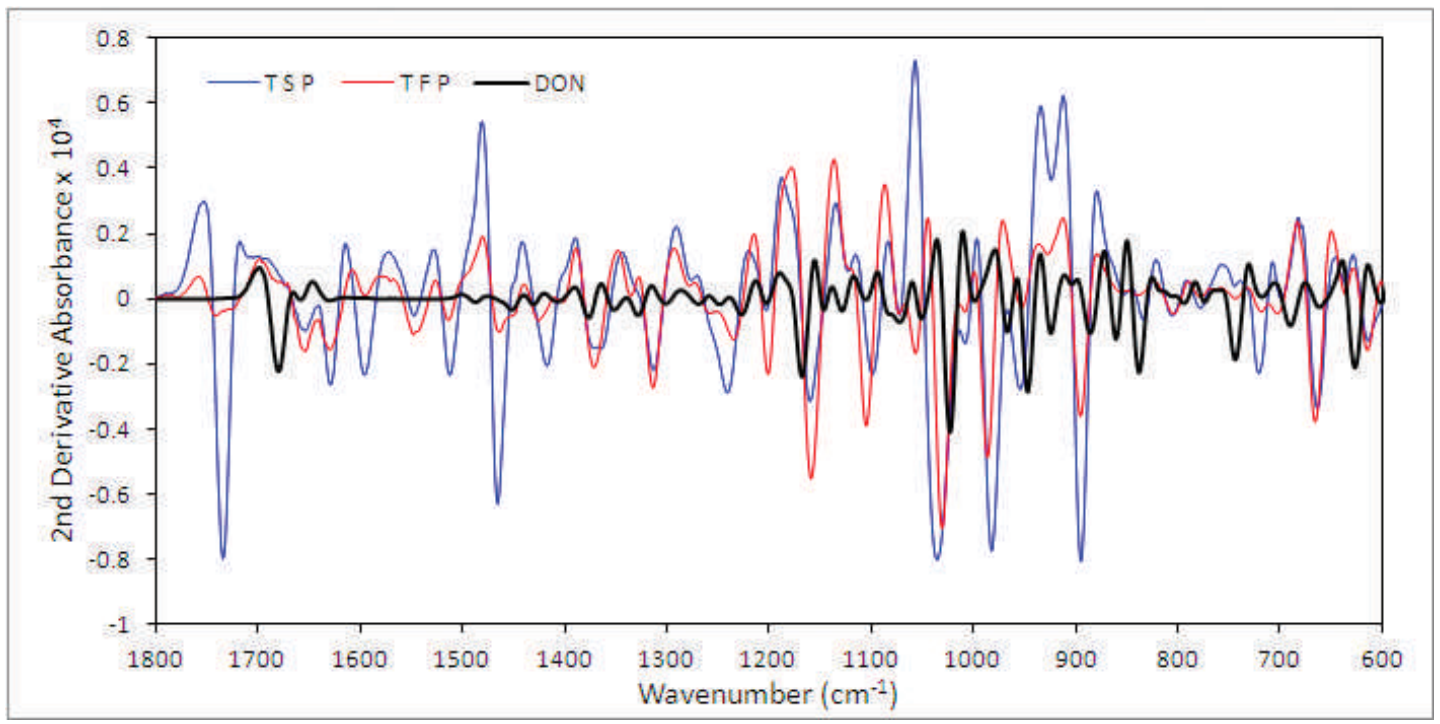

FIG. 9. Second-derivative mid-IR spectra of Tomahawk sound pericarp (TSP) and Fusarium-damaged pericarp (TFP) compared with the DON spectrum.

Fusarium-damaged tissues from the kernel surface may be modified by the presence of DON in the tissue matrix.

\section{CONCLUSION}

When mid-IR spectra of wheat pericarp and germ sections were examined, differences in certain mid-IR absorption bands were observed between sound and Fusarium-damaged kernels. The comparison of those spectral differences in relation to the mid-IR absorption bands of DON and Fusarium graminearum hyphae showed some consistent differences across two varieties tested, providing evidence to suggest that these specific absorption differences between sound and Fusariumdamaged kernel parts may be caused by the presence of DON or Fusarium graminearum hyphae in the tissues of Fusariumdamaged kernels. These results suggest that infrared spectros- copy can detect DON that is found in high concentrations on the surface tissues of Fusarium-damaged wheat kernels.

1. D.W. Parry, P. Jenkinson, L. McLeod. "Fusarium ear blight (scab) in small grain cereals: a review". Plant Pathol. 1995. 44: 207-238.

2. J.P.F. D'Mello, C.M. Placinta, A.M.C. Macdonald. "Fusarium mycotoxins: a review of global implications for animal health, welfare and productivity". Anim. Feed Sci. Technol. 1999. 80: 183-205.

3. M. McMullen, R. Jones, D. Gallenberg. "Scab of wheat and barley: A reemerging disease of devastating impact". Plant Dis. 1997. 81: 1340-1348.

4. R. Dill-Macky. "Fusarium head blight (scab)". In: W.W. Bockus, R.L. Bowden, R.M. Hunger, W.L. Morrill, T.D. Murray, R.W. Smiley, editors. Compendium of Wheat Diseases and Pests. St. Paul, MN: American Phytopathological Society, 2010. Pp. 34-36.

5. P. Nicholson, E. Chandler, R.C. Draeger, N.E. Gosman, D.R. Simpson, M. Thomsett, A.H. Wilson. "Molecular tools to study epidemiology and toxicology of fusarium head blight of cereals". Eur. J. Plant Pathol. 2003. 109: 691-703.

6. L.K. Nielsen, J.D. Jensen, G.C. Nielsen, J.E. Jensen, N.H. Spliid, I.K. 
Thomsen, A.F. Justesen, D.B. Collinge, L.N. Jorgensen. "Fusarium Head Blight of Cereals in Denmark: Species Complex and Related Mycotoxins". Phytopathology. 2011. 101: 960-969.

7. P. Koch. "State of the art of trichothecene analysis". Toxicol. Lett. 2004 153: 109-112.

8. M. Schollenberger, H.T. Jara, S. Suchy, W. Drochner, H.M. Muller "Fusarium toxins in wheat flour collected in an area in southwest Germany". Int. J. Food Microbiol. 2002. 72: 85-89.

9. A.E. Desjardins, T.M. Hohn, S.P. McCormick. "Tricothecene biosynthesis in Fusarium species: Chemistry, genetics and significance". Microbiol. Rev. 1993. 57: 595-604.

10. M.O. Moss, U. Thrane. "Fusarium taxonomy with relation to tricothecene formation". Toxicol. Lett. 2004. 153: 23-28.

11. H.E. Hallen-Adams, N. Wenner, G.A. Kuldau, F. Trail. "Deoxynivalenol biosynthesis-related gene expression during wheat kernel colonization by Fusarium graminearum". Phytopathology. 2011. 101: 1091-1096.

12. Z. Kang, H. Buchenauer. "Immunocytochemical localization of fusarium toxins in infected wheat spikes by Fusarium culmorum". Physiol. Mol. Plant Pathol. 1999. 55: 275-288.

13. Z. Kang, H. Buchenauer. "Studies on the infection process of Fusarium culmorum in wheat spikes: Degradation of host cell wall components and localization of trichothecene toxins in infected tissue". Eur. J. Plant Pathol. 2002. 108: 653-660.

14. D.B. Bechtel, L.A. Kaleikau, R.L. Gaines, L.M. Seitz. "The effects of Fusarium graminearum infection on wheat kernels". Cereal Chem. 1985. 62: 191-197.

15. H. Jackowiak, D. Packa, M. Wiwart, J. Perkowski. "Scanning electron microscopy of Fusarium damaged kernels of spring wheat". Int. J. Food Microbiol. 2005. 98: 113-123.

16. J. Schnurer. "Distribution of fungal biomass among fine bran, course bran, and flour from wheat stored at four different moisture levels". Cereal Chem. 1991. 68: 434.

17. D.M. Trigo-stockli, C.W. Deyoe, R.F. Satumbaga, J.R. Pedersen "Distribution of deoxynivalenol and Zearalenone in milled fractions of wheat". Cereal Chem. 1996. 73: 388-391.

18. S.N. Wegulo, F.E. Dowell. "Near-infrared versus visual sorting of Fusarium-damaged kernels in winter wheat". Can. J. Plant Sci. 2008. 88: 1087-1089.

19. J.S. Shenk, J.J. Workman, M.O. Westerhaus. "Application of NIR spectroscopy to agricultural products". In: D.A. Burns, E.W. Ciurczak, editors. Handbook of Near-Infrared Analysis. New York: Marcel Dekker, Inc., 1992. Pp 383-431.

20. K.H.S. Peiris, M.O. Pumphrey, Y. Dong, E.B. Maghirang, W. Berzonsky,
F.E. Dowell. "Near-Infrared spectroscopic method for identification of Fusarium head blight damage and prediction of deoxynivalenol in single wheat kernels". Cereal Chem. 2010. 87: 511-517.

21. G. Kos, R. Krska, H. Lohninger, P.R. Griffiths. " A comparative study of mid-infrared diffuce reflection (DR) and attenuated total reflection (ATR) spectroscopy for the detection of fungal infection on RWA2-corn". Anal. Bioanal. Chem. 2004. 378: 159-166.

22. J.B. Reeves III, S.R. Delwiche. "Determination of Protein in Ground Wheat Samples by Mid-Infrared Diffuse Reflectance Spectroscopy”. Appl. Spectrosc. 1997. 51: 1200-1204.

23. J.M. Olinger, P.R. Griffiths. "Effects of Sample Dilution and Particle Size/ Morphology on Diffuse Reflection Spectra of Carbohydrate Systems in the Near- and Mid-Infrared. Part I: Single Analytes”. Appl. Spectrosc. 1993. 47: 687-694.

24. P.R. Shewry, R. D’Ovido, D. Lafiandra, J.A. Jenkins, E.N.C. Mills, F. Bekes. "Wheat grain proteins". In: K. Khan, P.R. Shewry, editors. Wheat Chemistry and Technology. St. Paul, MN: AACC International, 2009. Pp 223-298.

25. J.M. Fang, P.A. Fowler, J. Tomkinson, C.A.S. Hill. "The preparation and characterization of chemically modified potato starches". Carbohydr. Polym. 2002. 47: 245-252.

26. B. Stone, M.K. Morell. "Carbohydrates". In: K. Khan, P.R. Shewry, editors. Wheat Chemistry and Technology. St. Paul, MN: AACC International, 2009. Pp 299-362.

27. L.R. Barran, E.F. Schneider, P.J. Wood, C. Madhosingh, R.W. Miller. "Cell wall of Fusarium sulphureum; I. Chemical composition of the hyphal wall". Biochim. Biophys. Acta. 1975. 392: 148-158.

28. G. Socrates. Infrared and Raman Characteristics Group Frequencies. Chichester, UK: John Wiley, 2001. 3rd ed.

29. J.C. Young, D.E. Games. "Analysis of Fusarium mycotoxins by gas chromatography-Fourier transform infrared spectroscopy". J. Chromatogr. A. 1994. 663: 211-218.

30. G.T. Reedy, M.M. Mossoba. "FTIR and hyphenated FTIR Techniques for the Analysis of Food". In: M.M. Mossoba, editor. Spectral Methods in Food Analysis. New York: Marcel Dekker, Inc., 1999. pp. 325-396.

31. H. Susi, D.M. Byler. "Protein structure by Fourier transform infrared spectroscopy: second derivative spectra". Biochem. Biophys. Res. Commun. 1983. 115: 391-397.

32. H. Susi, D.M. Byler. "Resolution-enhanced fourier transform infrared spectroscopy of enzymes". Methods Enzymol. 1986. 130: 290-311.

33. K.H.S. Peiris, M.O. Pumphrey, Y. Dong, F.E. Dowell. "Fusarium Head Blight Symptoms and Mycotoxin Levels in Single Kernels of Infected Wheat Spikes". Cereal Chem. 2011. 88: 291-295. 